\title{
基于二元衍射面的长波无热化光学系统设计
}

\author{
张发平, 张华卫 \\ (四川长虹电子科技有限公司, 四川 绵阳 621000)
}

\begin{abstract}
摘要: 仅用 3 片透镜, 设计了一款匹配 $640 \times 512$ 氧化钥非制冷长波焦平面探测器的红外系统。该系 统焦距为 $100 \mathrm{~mm}, F$ 数为 1.1 , 总长为 $107 \mathrm{~mm}$, 工作波段范围为 $8 \sim 12 \mu \mathrm{m}$, 引入一片二元衍射面实 现无热化温度补偿功能。利用 ZEMAX 进行仿真设计, 结果表明: 在 $-40^{\circ} \mathrm{C} \sim+60^{\circ} \mathrm{C}$ 温度范围内, 系 统奈奎斯特频率 (30 $\mathrm{lp} / \mathrm{mm})$ 处 MTF 均达到 0.49 , 接近衍射极限。该系统具有焦距较长, 相对孔径 大、全视场像质优良、温度适应良好、二元面衍射效率高、易于加工和结构紧凑的特点。
\end{abstract}

关键词: 二元衍射面; 无热化; 红外系统; 结构紧凑

中图分类号：TN216 文献编识码：A 文章编号：1001-8891(2020)01-0025-05

\section{Design of Long-wave Athermal Optical System Based on Binary Diffraction Surface}

\author{
ZHANG Faping, ZHANG Huawei \\ (Sichuan Changhong Electronics Technology Development Co., Ltd., Mianyang 621000, China)
}

\begin{abstract}
Using only three lenses, an infrared system matching $640 \times 512$ uncooled vanadium oxide long-wave focal plane detector was designed. The system has a focal length of $100 \mathrm{~mm}$, an F-number of 1.1, a total length of $107 \mathrm{~mm}$, and a working band range of 8-12 $\mu \mathrm{m}$. A binary diffraction surface was introduced to realize the non-thermal temperature compensation function. The simulation design using Zemax shows that the modulation transfer function (MTF)value at the Nyquist frequency $(30 \mathrm{lp} / \mathrm{mm})$ of the system reached 0.49 in the temperature range of $-40^{\circ} \mathrm{C}-+60^{\circ} \mathrm{C}$, which is close to the diffraction limit. The system has the characteristics of a long focal length, large relative aperture, good image quality in the full field of view, suitable temperature adaptation, high binary diffraction efficiency, easy processing, and compact structure.
\end{abstract}

Key words: binary diffraction surface, athermalization, infrared system, compact structure

\section{0 引言}

在红外相机实际使用过程中, 当环境温度变化 时, 由于热胀冷缩和折射率温变等因素, 透镜的折射 率、曲率半径、轴向尺寸、径向半径以及镜筒尺寸等 都会发生相应变化, 从而导致系统出现严重的像差和 离焦现象, 影响红外相机成像质量。因此, 必须采用 温度补偿措施来适应宽温度范围的环境使用条件。概 括来说, 温度补偿主要有两种方式: 一种是调焦式温 度补偿, 包括电子主动和机械被动式调焦。即当环境 温度发生变化时, 采用人眼观察或温度传感器反馈的 方式, 手动或自动调节某组镜片轴向位置, 实现温度 补偿; 另外一种就是光学被动无热化温度补偿。该方 式利用不同热系数材质的透镜和镜筒进行综合设计, 保证在宽范围温度环境下, 免调焦而不影响成像质
量。显然, 由于要增加调节机构, 采用调焦温度补偿 这种方式会导致系统重量和尺寸增加。并且, 在低温 差 (目标/背景) 条件下, 自动调焦速度会变得十分缓 慢, 调焦时间长, 甚至出现调焦不准的情况, 严重影 响实际使用效果, 所以光学无热化镜头设计成为了光 学系统研究的重要方向。

光学无热化设计除了采用多种材料组合消除高 低温带来的像差和离焦影响外, 还可以采用二元衍射 面消除热差。二元衍射元件具有独特的温度特性, 在 合理光焦度分配下, 可以用较为简单的结构实现温度 自动补偿 ${ }^{[1]}$ 。所以, 随着衍射面加工工艺技术的发展, 采用二元衍射面消热差的设计也变得十分常见。另 外, 随着长波非制冷探测器国产化, 相应非制冷红外 镜头的需求也变得越来越旺盛，但市面上长波非制冷 光学无热化镜头产品却仍然较少, 特别是焦距较长的 
无热化镜头比较稀缺。相关文献表明 ${ }^{[2-5]}$ : 目前长波无 热化镜头焦距一般不到 $100 \mathrm{~mm}$, 总长/焦距比大, 镜 片数较多。为此, 本文基于二元衍射面消热差原理, 针对市面典型的 $640 \times 512$ 非制冷面阵探测器, 仅使 用 3 片透镜, 设计了 $100 \mathrm{~mm}$ 焦距, 总长仅 $107 \mathrm{~mm}$ 的紧凑型、大相对孔径长波无热化系统。同时, 分析 了衍射面的参数和衍射效率, 为实际加工提供数据参 考。

\section{1 二元衍射面消热差原理}

根据薄透镜模型, 折射元件的光热膨胀系数 $x_{f, \mathrm{r}}$ 可用下式表示 ${ }^{[6]}$ :

$$
x_{f, \mathrm{r}}=a_{\mathrm{g}}-\frac{1}{n-n_{0}}\left(\frac{\mathrm{d} n}{\mathrm{~d} T}-n \frac{\mathrm{d} n_{0}}{\mathrm{~d} T}\right)
$$

式中: $a_{\mathrm{g}}$ 为材料线膨胀系数; $n 、 n_{0}$ 分别为透镜材料 和介质折射率; $\mathrm{d} n / \mathrm{d} T$ 为透镜材料的折射率温度系数。

对于二元衍射面, 其光热膨胀系数 $x_{f, \mathrm{~d}}$ 如下:

$$
x_{f, \mathrm{~d}}=2 a_{\mathrm{g}}-\frac{1}{n_{0}} \frac{\mathrm{d} n_{0}}{\mathrm{~d} T}
$$

由式(2)可知, 二元衍射面的光热膨胀系数只与材 料线膨胀系数和介质折射率有关, 与材料的折射率无 关。一般来说, 红外材料的 $\mathrm{d} n / \mathrm{d} T$ 都很大, 其光热膨 胀系数 $x_{f, r}$ 为负, 而二元衍射面光热膨胀系数 $x_{f, r}$ 始终 为正 ${ }^{[7]}$ 。因此, 通过合理组合, 可以保证系统消热差。

目前常见的长波材料温度特性和光学特性如表 1 所示 ${ }^{[8]}$ 。

表 1 长波材料温度和光学特性

Table 1 Optical and thermal parameters of long-wave infrared material

\begin{tabular}{lcll}
\hline Material & $\begin{array}{c}n \\
(@ 10 \mu \mathrm{m})\end{array}$ & $\begin{array}{c}\mathrm{d} n / \mathrm{d} t \\
\left(10^{-6} / \mathrm{K}\right)\end{array}$ & $\begin{array}{c}a_{0} \\
\left(10^{-6} / \mathrm{K}\right)\end{array}$ \\
\hline $\mathrm{Ge}$ & 4.0032 & 396 & 5.8 \\
Znse & 2.4065 & 100 & 8.54 \\
Zns & 2.1986 & 43.3 & 6.6 \\
IG5 & 2.6051 & 70 & 14 \\
IG6 & 2.7777 & 41 & 21.2 \\
\hline
\end{tabular}

在实际设计过程中, 往往根据系统特点选择适当 的材料组合来达到消热差的目的。一般情况下, 至少 采用两种材料组合才能达到设计要求。

\section{2 光学系统无热化方程}

无热化光学系统设计要满足光焦度分配、消色差 和消热差 3 个方程, 分别如下 ${ }^{[9]}$ :

$$
\begin{gathered}
\sum_{i=1}^{j} h_{i} \phi_{i}=\phi \\
\left(\frac{1}{h_{1} \phi}\right)^{2} \sum_{i=1}^{j} h_{i}^{2} \phi_{i} \theta_{i}=0 \\
\left(\frac{1}{h_{1} \phi}\right)^{2} \sum_{i=1}^{j} h_{i}^{2} \phi_{i} \chi_{i}=\sum_{i=1}^{j} a_{i} L_{i}
\end{gathered}
$$

式中: $h_{i} 、 \phi_{i} 、 \theta_{i}$ 和 $\chi_{i}$ 分别为各透镜组近轴光线高度、 光焦度、色差系数和热差系数; $h_{1}$ 为第一个透镜近轴 光线高度; $\phi$ 为系统总光焦度; $a_{i}$ 为各部分镜筒材料的 线膨胀系数; $L_{i}$ 为各部分镜筒长度。

\section{3 设计实例}

\section{1 设计参数}

针对国内典型的 $640 \times 512$ 氧化钒非制冷探测器, 设计一款紧凑型较长焦距的长波无热化红外系统, 具 体参数如表 2 所示。

表 2 光学系统参数

Table 2 Optical system parameters

\begin{tabular}{ll}
\hline Wavelength band & $8 \sim 12 \mu \mathrm{m}$ \\
Detector resolution & $640 \times 512$ \\
Pixel size & $17 \mu \mathrm{m}$ \\
Focal length & $100 \mathrm{~mm}$ \\
Overall length & $107 \mathrm{~mm}$ \\
F numbers & 1.1 \\
Optical transmissivity & $\geqslant 80 \%$ \\
Working temperature & $-40^{\circ} \mathrm{C} \sim+60^{\circ} \mathrm{C}$ \\
\hline
\end{tabular}

\section{2 设计结果}

首先根据式(3) (5)计算出理想透镜的光焦度分 配, 然后将理想透镜换成实际材料的厚透镜后再利用 光学设计软件优化像差和消热差, 最终设计得到的系 统仅由两个镜组, 共 3 片透镜组成。前后两个透镜组 采用 “正十负” 光焦度分配方式, 并将入瞳设计在第 一个面, 以减少系统口径。综合考虑常见长波材料的 温度和色散特性, 系统前组采用折射率最高、消色差 能力强的单晶锗与低折射率的 IG6 两种材料相配合消 色差。由于单晶锗折射率温度系数 $\left(396 \times 10^{-6} \mathrm{~K}^{-1}\right)$ 较大, 因此, 在前组单晶锗镜片上引入一个二元衍射 面, 既可以提升色差消除效果, 又可以有效消除宽温 差带来的离焦影响。镜筒材料选择常见的铝合金, 其 热膨胀系数为 $23.6 \times 10^{-6} \mathrm{~K}^{-1}$, 与透镜配合实现光学 被动无热化。另外, 在后组单晶锗透镜上采用一个偶 次非球面校正剩余像差, 以保证系统成像质量。尽量 优化系统结构参数, 使得整个光学系统总尺寸不超过 
$\phi 93 \mathrm{~mm} \times L 107 \mathrm{~mm}$, 总长/焦距比仅为 1.07 , 结构紧凑。 结构布局如图 1 所示。

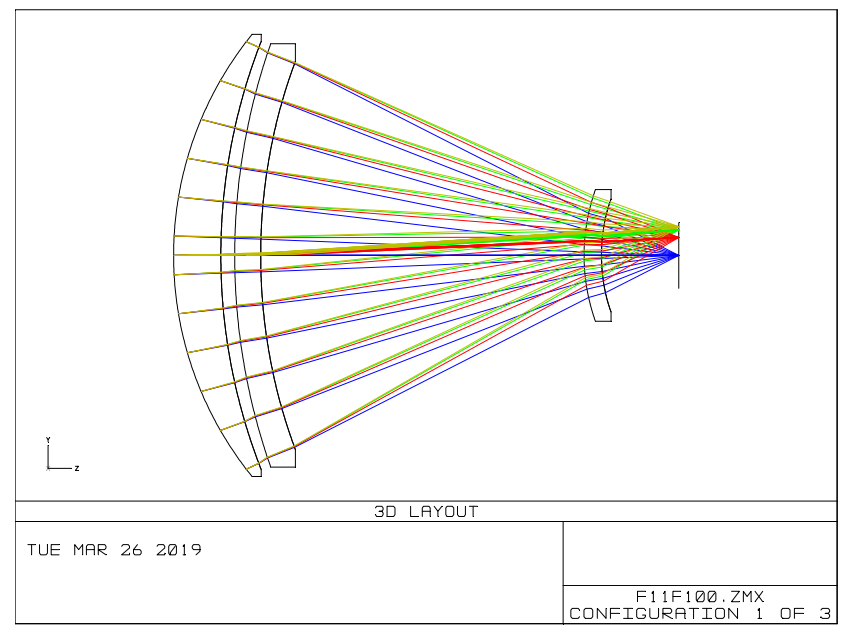

图 1 光学系统结构图

Fig.1 Schematic of optical system structure

\section{3 成像质量评价}

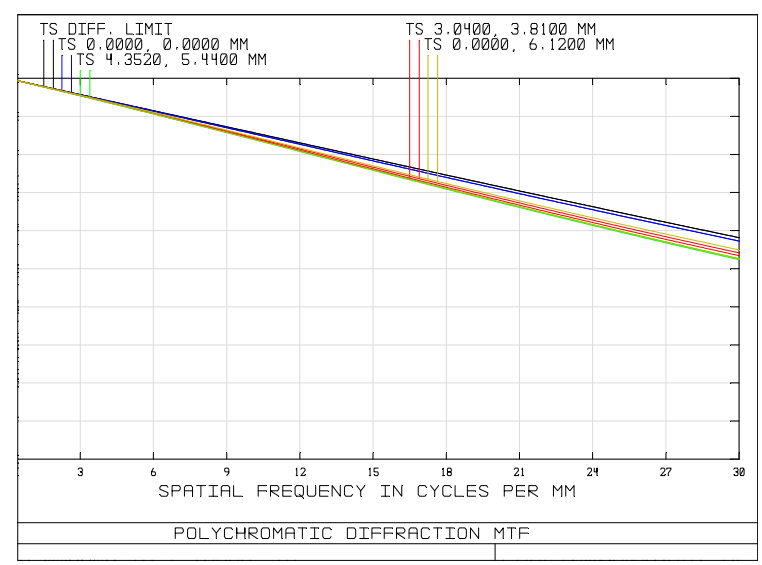

(a) $+20^{\circ} \mathrm{C} \mathrm{MTF}$
调制传递函数 (MTF) 和弥散斑是衡量光学系统 成像质量的重要指标, 由图 2、图 3 和表 3 可知, 在 系统奈奎斯特频率 $(30 \mathrm{lp} / \mathrm{mm})$ 处各视场的 MTF 值均 达到 0.49 , 接近衍射极限; 弥散斑均方根半径都小于 艾利斑半径 $13.4 \mu \mathrm{m}$; 最大场曲小于 $0.1 \mathrm{~mm}$, 最大畸 变小于 $1.6 \%$; 最大离焦量为 $13.54 \mu \mathrm{m}$, 小于系统焦深 $24.2 \mu \mathrm{m}$ 。综上, 表明该系统在 $-40^{\circ} \mathrm{C} \sim+60^{\circ} \mathrm{C}$ 范围内 成像质量优异, 无热化效果良好。

\section{4 衍射面加工参数}

受限于车床加工技术, 二元衍射面面型结构的复 杂程度对加工可行性及成本起着决定性的影响。因 此, 在利用光学软件仿真完衍射面相位函数后, 需要 重点分析该二元衍射面的加工难易程度。考虑加工因 素和衍射效率, 取位相函数阶数为 3 , 衍射级次为 1 , 则位相函数可具体表达为式如下 ${ }^{[10]}$ :

$$
\left|A_{1} \rho^{2}+A_{2} \rho^{4}+A_{3} \rho^{6}\right|=2 n_{\mathrm{r}} \pi \quad\left(n_{\mathrm{r}}=1,2,3, \cdots\right)
$$

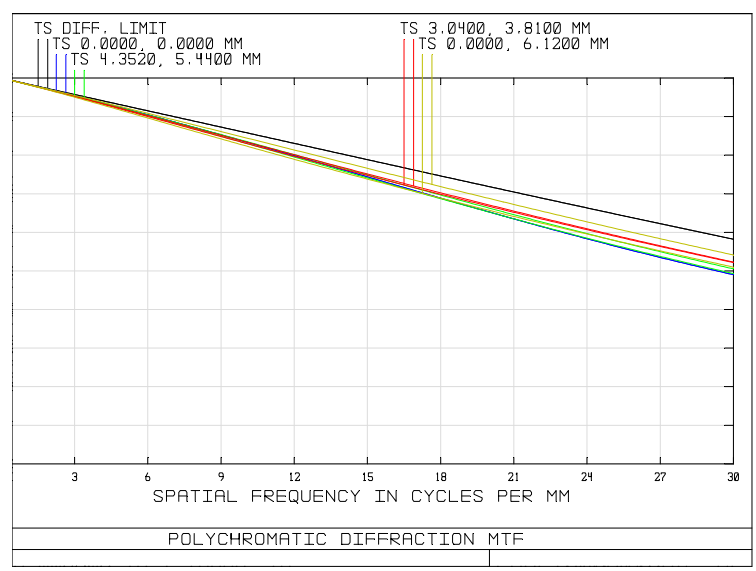

(b) $-40^{\circ} \mathrm{C}$ MTF

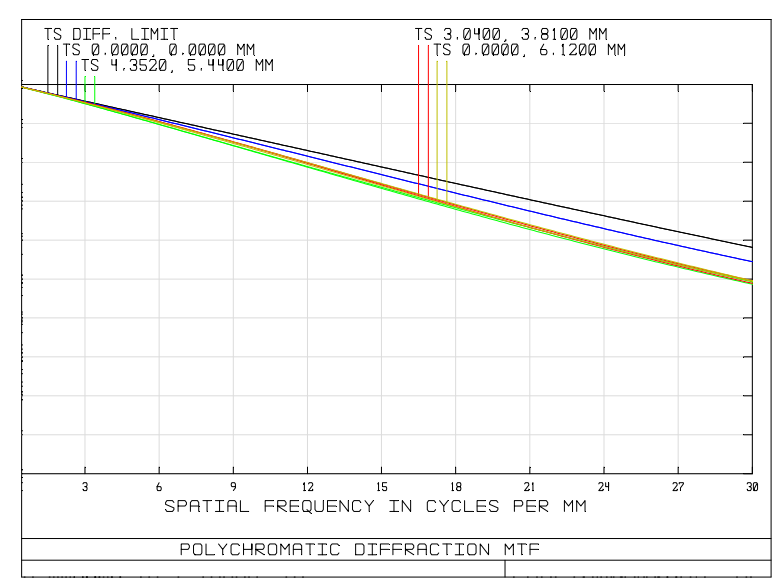

(c) $+60^{\circ} \mathrm{C}$ MTF

图 2 系统在 $-40^{\circ} \mathrm{C} \sim+60^{\circ} \mathrm{C}$ 范围 $\mathrm{MTF}$

Fig.2 System MTF curves in the range of $-40^{\circ} \mathrm{C}-+60^{\circ} \mathrm{C}$ 


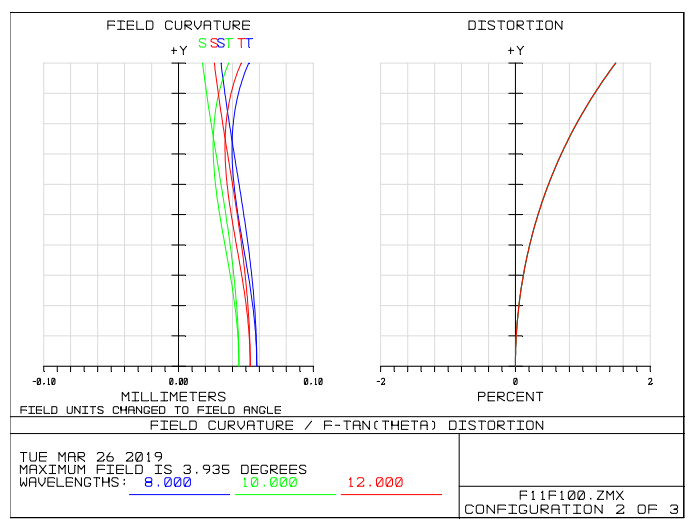

(a) $-40^{\circ} \mathrm{C}$

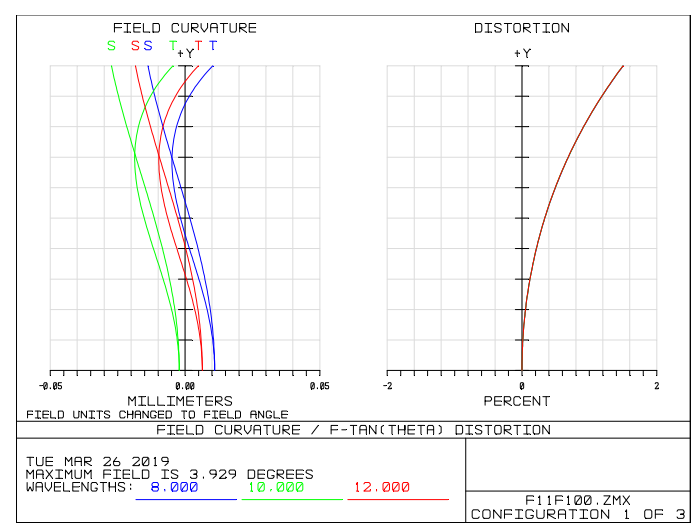

(b) $+20^{\circ} \mathrm{C}$

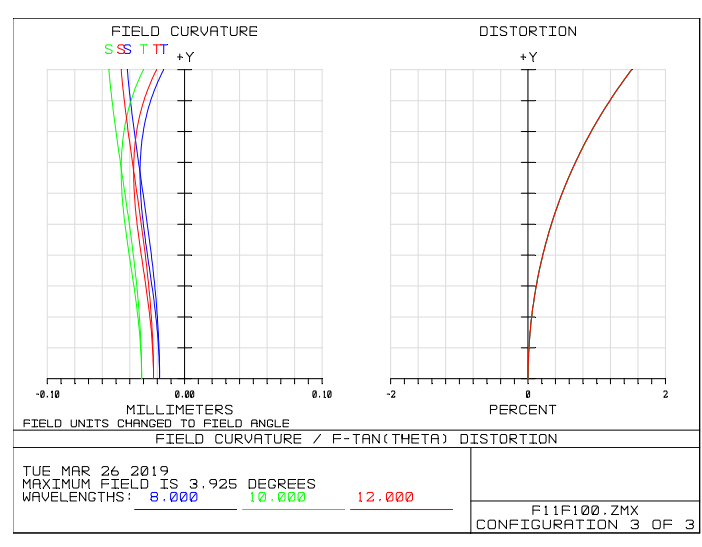

(c) $+60^{\circ} \mathrm{C}$

图 3 系统在 $-40^{\circ} \mathrm{C} \sim+60^{\circ} \mathrm{C}$ 场曲和畸变

Fig.3 System dist and field curves in the range of $-40^{\circ} \mathrm{C} \sim+60^{\circ} \mathrm{C}$

表 3 系统在 $-40^{\circ} \mathrm{C} \sim+60^{\circ} \mathrm{C}$ 范围弥散斑

Table 3 System spot diagram in the range of $-40^{\circ} \mathrm{C}-+60^{\circ} \mathrm{C}$

\begin{tabular}{llll}
\hline \multirow{2}{*}{ Spot diagram $/ \mu \mathrm{m}$} & \multicolumn{3}{c}{ Temperature } \\
\cline { 2 - 4 } & $-40^{\circ} \mathrm{C}$ & $+20^{\circ} \mathrm{C}$ & $+60^{\circ} \mathrm{C}$ \\
\hline 0 field & 9.386 & 2.492 & 6.675 \\
0.7 field & 11.906 & 4.758 & 7.409 \\
1.0 field & 8.487 & 6.268 & 12.996 \\
\hline
\end{tabular}

最大环带深度 $h_{\max }$ 由式(7)表示:

$$
h_{\text {max }}=\frac{2 \lambda_{\mathrm{s}} \lambda_{1}}{\left(n_{\text {mid }}-1\right)\left(\lambda_{\mathrm{s}}+\lambda_{1}\right)}
$$

式中: $\rho$ 为衍射面的环带半径; $n_{\mathrm{r}}$ 为环带数; $\lambda_{\mathrm{s}} 、 \lambda_{1}$ 分别为衍射波长上限和下限; $n_{\text {mid }}$ 为中心工作波长折 射率。

衍射效率对于二元衍射面来说是比较重要的物 理量, 它直接关系到光学系统总透过率, 影响系统总 的接收能量及作用距离。衍射效率和加工水平与加工 参数密切相关, 目前主要采用超精密数控金刚石单点 车削的方式加工二元衍射面。根据研究表明, 采用车 削的方式加工二元衍射面时, 其衍射效率主要受如下 3 个方面的因素影响 ${ }^{[11]}$ : 一是车削面型环带过度处遮
挡效应导致的衍射效率损失 $L_{1}$; 二是波段内平均衍射 效率损失 $L_{2}$; 三是表面粗䊁程度造成 TIS 散射量的衍 射效率损失 $L_{3}$, 以上 3 个方面的影响因素可以分别由 式(8)、(9)、(10)表示:

$$
\begin{gathered}
L_{1}=\frac{4}{D} \sqrt{\frac{2 h_{\text {max }} R_{\mathrm{T}}}{n_{\text {rtoal }}}} \sum_{1}^{n_{\text {toak }}} n_{\mathrm{r}} \\
L_{2}=\left(\frac{\pi\left(\lambda_{1}-\lambda_{\mathrm{s}}\right)}{6 \lambda_{\text {mid }}}\right)^{2} \\
L_{3}=\left(\frac{4 \pi \times 0.4 \times d^{2}}{8 \times R_{\mathrm{T}} \times \lambda_{\text {mid }}}\right)^{2}
\end{gathered}
$$

式中: $D$ 表示衍射面的有效通光口径; $\lambda_{\text {mid }}$ 为工作中 心波长; $n_{\mathrm{rtoal}}$ 为衍射元件总周期个数; $R_{\mathrm{T}}$ 为球形切削 刀具刀尖半径; $d$ 为车削刀给进量。因此, 在二元衍 射面的设计和加工过程中, 不仅要考虑衍射面本身的 参数, 还要考虑采用合适的加工参数, 以保证较大的 衍射效率。

根据现有的金刚石单点车削加工水平和设计需 
求，本设计中衍射面各个参数的取值如表 4 所示。 表 4 衍射面加工参数

Table 4 Machining parameters of diffractive surface

\begin{tabular}{lcc}
\hline & \multicolumn{1}{c}{ Parameter } & Value \\
\hline \multirow{2}{*}{$\begin{array}{c}\text { Phase function } \\
\text { coefficient }\end{array}$} & $A_{1}$ & -0.071 \\
\cline { 2 - 3 } & $A_{2}$ & $3.561 \times 10^{-7}$ \\
\cline { 2 - 3 } Wavelength & $A_{3}$ & $-6.834 \times 10^{-11}$ \\
band & Upper and lower limits & $\lambda_{\mathrm{s}}=8 \mu \mathrm{m}$, \\
& Central working wavelength & $\lambda_{\mathrm{I}}=12 \mu \mathrm{m}$ \\
\hline Refractive index of central working wavelength & $\lambda_{\text {mid }}=10 \mu \mathrm{m}$ \\
\hline Radius of spherical cutting edge & $R_{\mathrm{T}}=50.0032$ \\
\hline Feed size of turning tool & $d=0.3 \mu \mathrm{m}$ \\
\hline
\end{tabular}

利用 Matlab 编程迭代求解方程(6)和(7), 得到最 大环带周期数为 19 (环带周期见图 4), 最大环带深 度为 $3.33 \mu \mathrm{m}$, 最小环带间隔为 $1.106 \mathrm{~mm}$, 二元衍射 面设计在单晶锗材料上, 加工难度较低。由式(8) (10) 估算总的衍射效率约为 $92 \%$, 衍射效率较高, 其余每 个镜面透过率按 98\%估算, 则整个系统透过率约为 $83 \%$, 符合设计要求。

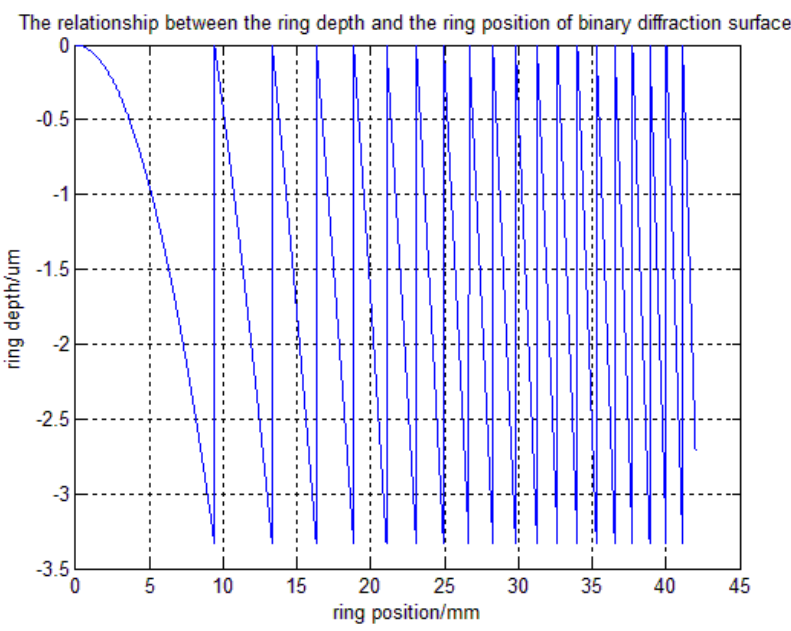

图 4 二元衍射面的环带周期

Fig.4 Ring periods of binary diffraction surface

\section{4 结语}

本文根据二元衍射面消热差理论, 基于 $640 \times 512$ 焦平面长波非制冷探测器, 设计出一种大相对孔径、 较长焦距、低总长/焦距比的光学无热化红外系统。该 系统在 $-40^{\circ} \mathrm{C} \sim+60^{\circ} \mathrm{C}$ 范围内, MTF 值接近衍射极 限, 像质优良, 温度适应性好。通过 Matlab 仿真计算, 所采用的二元衍射面具有环带数较少, 环带间隔大, 衍射效率高, 易加工的特点。由于衍射面的引入, 克 服了紧凑型、大相对孔径、较长焦距光学系统难以实
现光学无热化的难点, 为红外监控、警戒的非制冷光 学系统设计提供一个参考。

\section{参考文献:}

[1] Behrmann G P , Bo Wen J P. Influence of temperature on diffractive lens performance[J]. App. Opt., 1993, 32(14): 2583-2489.

[2] 王远方舟, 白玉环, 贾钰超, 等. 一种长波红外光学消热差系统设计 [J]. 红外技术, 2012, 34(9): 531-534.

WANG Yuanfangzhou, BAI Yuzhuo, JIA Yuchao, et al. A design of FIR athermal system[J]. Infrared Technology, 2012, 34(9): 531-534..

[3] 陈潇. 大面阵长波红外光学无热化镜头的设计 [J]. 红外技术, 2018, 40(11): 1061-1064.

CHEN Xiao. Design of long-wavelength infrared athermalization lens for large-array detector[J]. Infrared Technology, 2018, 40(11): 1061-1064.

[4] 史浩东, 张新, 曲贺盟, 等. 基于硫系玻璃的大相对孔径红外消热差 光学系统设计 [J]. 光学学报, 2015, 35(6): 0622002.1-0622002.7.

SHI Haodong, ZHANG Xin, QU Hemeng, et al. Design of large relative aperture Infrared athermalized optical system with chalcogenide glasses[J]. Acta Optica Sinica, 2015, 35(6): 0622002.1-0622002.7.

[5] 张婉怡. 红外折衍混合摄远光学系统无热化设计[J]. 应用光学, 2017, 38(1): $12-18$.

ZHANG Wanyi. Athermalization design of infrared refractive diffractive telephoto objective[J]. Journal of Applied Optics, 2017, 38(1): 12-18.

[6] 白瑜, 杨建峰, 马小龙, 等. 长波红外光学系统无热化设计[J]. 红外技 术, 2008, 30(10): 583-585.

BAI Yu, YANG Jianfeng, MA Xiaolong, et al. Athermalization of long-wavelength infrared optical system[J]. Infrared Technology, 2008, 30(10): 583-585.

[7] 徐大维, 向阳, 王建, 等. 折衍混合车载红外镜头无热化设计 [J]. 红外 技术, 2011, 33(8): 461 .

XU Daxiong, XIANG Yang, WANG Jian, et al. Design on athermal refractive-diffractive hybrid vehicular infraredlens $[\mathrm{J}]$. Infrared Technology, 2011, 33(8): 461.

[8] 孙爱平, 龚杨云, 陈忠, 等. 大孔径、大视场辅助驾驶仪红外镜头无热 化设计[J]. 红外技术, 2013, 35(10): 618 .

SUN Aiping, GONG Yangyun, CHEN Zhong, et al. Athermal design of a large-aperture, wide-field assisting pilot infrared lens[J]. Infrared Technology, 2013, 35(10): 618.

[9] HUANG Qiu, CHEN Yiqing, GAO Zhifeng, et al. Athermalization and test validation of infrared imaging system[C]/SPIE, 2009, 7383: 73830P.

[10] 农文捷, 金宁. 二元光学工艺参数计算 [J]. 红外技术, 2004, 26(6): 13-16.

NONG Wenjie, JIN Ning. Evaluation of binary optics manufacturing parameters[J]. Infrared Technology, 2004, 26(6): 13-16.

[11] 王鹏. 衍射光学元件设计及金刚石单点车削技术的研究[D]. 长春: 长春理工大学, 2006: 23-30.

WANG Peng. Research on design and process parameters of diamond turning of diffractive optical[D]. Changchun: Changchun University of Science and Technology Optical engineering, 2006: 23-30. 